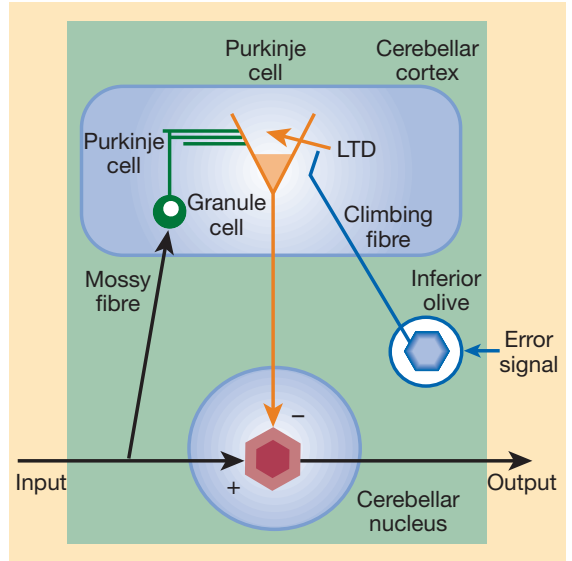

Figure 2 Basic structure of the neuronal circuitry in the cerebellum. A single functional 'unit' is shown. Mossy-fibre signals provide inputs to the unit and neurons in the cerebellar nucleus generate outputs. Climbing-fibre signals represent errors, reorganizing internal connections within the unit to modify its input-output relationship. LTD, long-term depression. (Adapted from ref. 3.)

the realized movement is similar to the 'instruction' movement. The instruction may also be converted to command signals by the cerebellum, instead of by the cerebral cortex.

Even without such feedback, this pathway could produce a movement that closely matches the movement received as an instruction. For this to happen, however, the cerebellum would need to contain the inverse of the dynamics or kinematics of the hand/arm system - in other words, the cerebellum would need to contain an inverse model of the hand/arm system. The model is formed and updated through learning, by referring to the errors between the intended and performed movements, as detected by the motor and related areas of the cerebral cortex (feedback-error learning) ${ }^{2}$.

To test this hypothetical prediction, Imamizu et al. ${ }^{1}$ asked a human subject lying in a functional magnetic resonance imaging (fMRI) scanner to manipulate a computer mouse. During the so-called 'baseline' period, the subject followed a moving square target with a cross-hair cursor on a screen. Then, during the test, the position of the cursor was rotated $120^{\circ}$ around the centre of the screen to provide a new mouse 'condition'. At first, during the test period, large regions of the cerebellum were significantly activated compared with their activity during the baseline period. But this activation decreased after repeated test trials, in parallel with a reduction of the tracking errors. However, certain restricted sub-regions of the brain (near the posterior superior fissure) continued to be activated. The authors propose that this remaining activity represents an internal model that is formed during the repeated test trials. This model defines the new relationship between movement of the cursor and of the mouse.

The internal-model concept for motor learning is reasonable if we consider the known cellular mechanisms of the cerebellum. The cerebellum has a compartmentalized structure that consists of numerous small, functional units, each of which contains a neuronal network, composed of Purkinje and other neurons, organized in a geometrically beautiful way (Fig. 2). Each unit can change its input-output relationship through learning, which is driven by error signals conveyed to the Purkinje cells by a unique input structure, the climbing fibres. Climbing-fibre signals induce longterm depression (LTD) of transmission from a major input to the Purkinje cells, via the mossy fibre-parallel fibre pathway ${ }^{3}$. LTD depresses those synapses between the parallel fibres and Purkinje cells that are involved in making errors, thereby reorganizing internal connections towards a reduction of the errors. Through this learning mechanism a cerebellar unit could modify its inputoutput relationship until it represents the model required for precise control.

A crucial question is whether an increase in local blood flow in the cerebellum, as detected by $\mathrm{AMRI}$, really represents the formation and maintenance of an internal model. If LTD is the only synaptic plasticity that underlies the learning mechanism of the cerebellar circuitry, the learning is unlikely to be accompanied by an increase in electric impulse discharges (which may be reflected as increased local blood flow). So the authors assume that excitatory and inhibitory synaptic transmissions to the Purkinje cells are facilitated, and that electric-impulse discharges of the Purkinje cells increase.

But in the complex neuronal circuitry of the brain there maybe other possibilities. For example, many chemical reactions underlie the induction of LTD, including the release of nitric oxide, which has a well-known action of relaxing blood capillaries. The next question to address experimentally is how not only the electric-impulse discharges, but also the complex chemical processes, contribute to increases in localblood flow in the cerebellum.

As neuroscientists strive to understand the molecular and cellular events that occur in neurons, effective technologies and methodologies for experimentally investigating computational and cognitive principles of the brain are still in short supply. The success of Imamizu et al. ${ }^{1}$ in visualizing an internal model in the cerebellum should therefore provide tremendous encouragement for other researchers in the field.

Masao Ito is at the Brain Science Institute, RIKEN,

Wako, Saitama 351-0198, Japan.

e-mail:masao@mailsv.brain.riken.go.jp

1. Imamizu, H. et al. Nature 403, 192-195 (2000).

2. Kawato, M. \& Gomi, H. Biol. Cybern. 68, 95-103 (1992).

3. Ito, M. Trends Cog. Sci. 2, 313-321 (1998).
Daedalus

\section{Coal, air, fire and water}

Coal-mining is a costly, labour-intensive activity. Many attempts have been made to extract at least some of the energy of coal in situ, by underground gasification. Daedalus is now updating this idea.

A coal mine, he points out, fills with water unless constantly pumped out. In a flooded mine the water is under hydrostatic pressure. At the bottom of a mine more than about $2.2 \mathrm{~km}$ deep, it would reach supercritical pressure. Heated, it could not boil, but would go to supercritical water - a powerful solvent and reaction medium. If it contained dissolved oxygen, organic materials including coal would burn spontaneously in it. So, says Daedalus, drill two holes down to a sufficiently deep coal seam, lower some sort of robot borer to drill a tunnel connecting the two holes, and fill the whole thing with water. Ignite the coal electrically or pyrotechnically, and pump aerated water down one shaft and up the other. The water flow will then deliver the products to the surface.

What will those products be? In the presence of excess fuel - such as a vast coal seam - supercritical combustion is a very partial business. Toluene, for example, burns in limited oxygen to benzoic acid. Coal will go to big aromatic or quinonoid molecules, with enough oxygen in them to dissolve in the water. The upcoming stream of hot water will boil as the hydrostatic pressure lessens. The steam will contain the more volatile organics, easily separable by fractionation, ideally by stepwise expansion in a steam engine. Bigger molecules will stay in the water; some will crystallize out as it cools. So the mine will yield steam power, plus a stream of useful fuels and chemical feedstocks. Sulphur dioxide in the water will be processed to useful sulphuric acid, and solid ash will mainly stay underground.

A supercritical mine will access a vast amount of coal. The initial narrow tunnel will soon widen laterally as it eats the seam away from between the rocky strata above and below it. As it widens, the tunnel will slowly collapse in the middle by subsidence, giving two tunnels. These will gradually move apart through the seam, scouring it out as subsidence propels them sideways. The vast coal reserves more than $2.2 \mathrm{~km}$ down, too deep for normal mining, will be accessible at last. David Jones

The Further Inventions of Daedalus (Oxford University Press), 148 past Daedalus columns expanded and illustrated, is now on sale. Special Nature offer: m.curtis@nature.com 